\title{
InformationBased Hierarchical Brain Organization/ Evolution from the Perspective of the Informational Model of Consciousness
}

\author{
Florin Gaiseanu* \\ Principal Senior Researcher, Science of Information and Technology Bucharest, Spain
}

*Corresponding author: Florin Gaiseanu, Principal Senior Researcher,

Science of Information and Technology Bucharest, Spain.

Received Date: March 20, 2020

Published Date: April 14, 2020

\begin{abstract}
s
Introduction: This article discusses the brain hierarchical organization/evolution as a consequence of the information-induced brain development, from the perspective of the Informational Model of Consciousness.

Analysis: In the frame of the Informational Model of Consciousness, a detailed info-neural analysis ispresented, concerning the specific properties/functions of the informational system of the human body composed by the Center of Acquisition and Storing of Information, Center of Decision and Command, Info-Emotional Center, Maintenance Informational System, Genetic Transmission System, Info Genetic Generator and Info-Connection center, in relation with the neuro-connected brain areas, with a special attention to the Info-Connection and its specific properties. Besides a meticulous analysis of the info-connections/neuro-functions of these centers, a special attention was paid to limbic/cingulate cortex activities. Defined as a trust/confidence center, additional features are highlighted in correlation with the activity of the anterior cingulate cortex, consisting in the intervention/moderation of amygdala emotional signals, conflicting opposite YES/NO data and error elimination in the favour of the organism equilibrium, the intervention in the certainty/uncertainty balance to select a suitable pro-life information (antientropic effect), in moderation of pain and in the stimulation of the empathic inter-human relations/communication. Representing the correspondence between the informational subsystems and the brain area map, it is shown that the up/down integration of information by epigenetic mechanisms and the down/ up evolution are correlated.
\end{abstract}

Results: The analysis of the functions of the anterior cingulate opens new gates of investigationsconcerning the involved intimate mechanisms at the level of cell microstructure, specifically on the compatibility with quantum assisted processes admitted by the Informational Model of Consciousness and the quantum-based models The discussion on the information integration/codification by epigenetic mechanisms shows that this process starts from the superior levels of brain conscious info-processing areas and progressively advances to the automatic/autonomic inferior levels of the informational system, under insistent/repetitive cues/stress conditions, pointing out an hierarchical functional/anatomical structure of the brain organization. Additional arguments are discussed, indicating that the down/up progressive scale representation is a suggestive illustration of the brain evolution, induced/assisted/determined by information, accelerated at humans by the antientropic functions of the Info-Connection center.

Conclusions: The hierarchical organization of the brain is a consequence of the integration process ofinformation, defining its development accordingly to the adaptation requirements for survival during successive evolution stages of the organism, information playing a determinant/key role.

Keywords: Information; Informational systems; Brain hierarchical organization/evolution; Information induced evolution

\section{Introduction}

In some recent articles it was shown that the human body activity could be assigned to two main categories, which were defined as poles or axes of the organism: information and matter[1]. The human body could be regarded as a bipolar info- matter structure, where information plays a key role for adaptation and survival [2,3]: on one hand the capture of information from environment is necessary for momentary adaptation, and on the other hand matter processing assures the body power and regeneration resources. The processing of the insistently 
transmitted information from the surrounding ambient by cues/ stress stimuli, or by learning and acquired abilities, is assimilate into the matter genetic structure of the body, and can be transferred to the next generation by means of epigenetic processes [4]. The intimate mechanisms of the transmission of information between and inside of the cells were explained also recently within the Informational Model of Consciousness, by information-assisted embodiment/disembodiment interactions [5]. The implication of such info-matter processes into the body activity and operation shows that information is a fundamental agent for adaptation and survival. Beside information, the circuit of matter, which can be defined as a metabolic axis of the organism, is responsible to sustain mainly the power/energetic/regeneration resources of the organism $[4,6]$.

As it was stipulated recently [7], information is a key for the life and life origin. This was however argued in the previous Draganescu's works, which founded/established a decisive milestone in the science of information in this domain, showing that information is not only a fundamental agent involved in the living, but also in the matter structuration $[8,9]$. According to his findings, the structuration of matter cannot be performed without the participation of information, as a deeply and actively involved factor, so the structuration process of matter is actually a consequence of the activation of ordinary (non structured deep layer) matter by information, which determines a sense and tendency for structuration. The following relation should be taken therefore into account: Unstructured Matter + information => Structured Matter. Moreover, a huge quantity of information is necessary to structure the living components to become an animate whole entity, according to the relation: Structured Matter + Information =>Living [9], as it was explained in detail earlier $[10,11]$. The human organism appears therefore to be a bipolar infomaterial structure with two poles/axes- information and matter, so the body itself can be defined as an informed-matter structure [10].

Starting from these outcomes and from the basic statements of the Informational Model of Consciousness recently reported [5], in this paper it is shown that information is not only a fundamental parameter to maintain the interaction with the environment and body itself, but also a key/determinant parameter for the brain hierarchical evolution and organization.

Basic Statements of the Informational Model of Consciousness and Info-Neural Analysis

\section{Basic Statements}

The Informational Model of Consciousness, shows that the architecture of the informational system of the human body is composed by seven main informational subsystems, assuring the management of its basic functions to comply with a fundamental objective: survival. Thisobjective regards the entire horizon of time:

(i) Reception/processing of genetic information from the parents (predecessors) as an Info-Genetic Generator (IGG).

(ii) Info-management of the matter-related necessities by means of the Maintenance Informational System (MIS) during the present existence time.

(iii) Info-genetic transmission of information for species survival in the future. However, the momentary or mediumterm adaptation for survival requires more engaged functions.

(iv) Processing of information for adaptation/correlation with the environment and body itself is assured by the Center of Decision and Command (CDC), responsible for the infooperability, info-processing/elaboration and transmission of the decision to the motor/execution elements.

(v) Center of Acquisition and Storing of Information (CASI), which keeps/offer the life experience data to CDC.

(vi) Info-Emotional System (IES) managing the emotional body reactions. A special informational activity is represented by the Info-Connection (IC) pole of the organism, helping/ assuring the life and body/mental equilibrium and normal informational work [10, 1-3]. This item will be furthermore discussed in detail latter(Figure 1).

\section{Info-neural analysis}

Each of these subsystems is connected with specific areas of the brain as represented schematically in the info-neural diagram in (Figure 1). IGG and GTS, mainly responsible for body development according to the age and the reproduction respectively, are mainly represented by the specific functions of hypophysis, situated to the lower part of the brain. With a size of a pea, the hypophysis has a very low weight, of only 0.5 grams. As small as it is, it plays an important role as a master gland, engaged together with hypothalamus to control the organism development and growth, managing the energy and metabolism, osmotic regulation in the body and water balance within the kidney, playing a role also in temperature regulation [11], functions specific of IGG. As it is to be expected, this also is involved in the control of the organism aging, as it was recently shown [12]. Hypothalamus is also involved in the sex/hormonal development and differentiated functions of female/male, influencing/determining distinct sexual activity and behaviour starting from the puberty age, by the control of the hypophysis secretion, while the basal ganglia seems to play a role in the inherited personality features and predispositions [13], specific of IGG. Defining the genetic transmitted support for the new generation as a matter-related information, within the Informational Model of Consciousness IGG is connected to the input genetic information. 


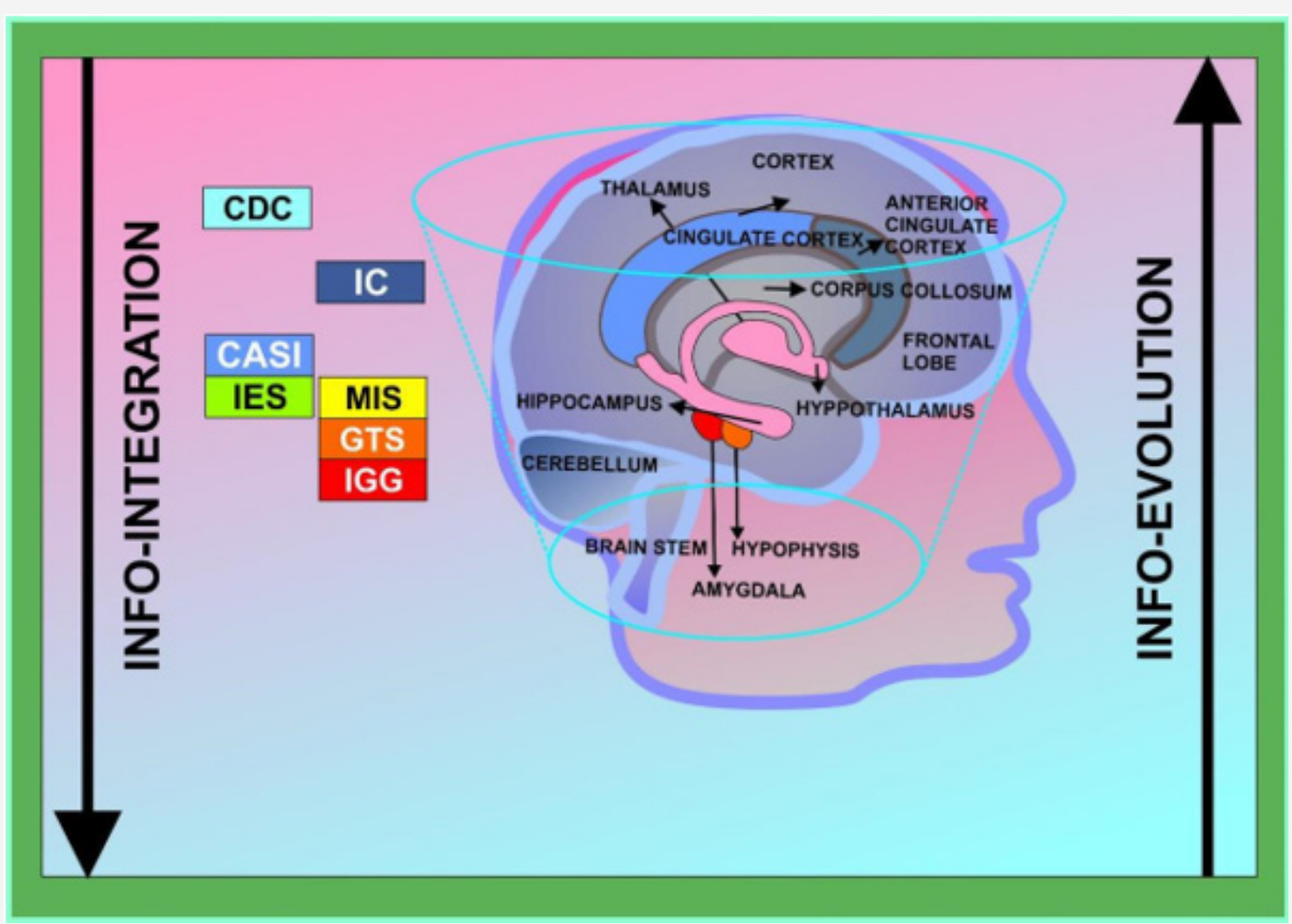

Figure 1: Info-neural diagram schematically representing the brain areas and the corresponding informational subsystems according to their main functions, allowing to observe the integration of the acquired information (left side) and the brain evolution/organization (right side).

Within GTS, the hypophysis and hypothalamus tandem control the reproduction functions, represented by the management of the activity of both masculine and female sex organs, particularly the pregnancy, childbirth and even pain relief during such process, the breastfeeding and the breast milk production. The informational communication with the specialized organs takes places by means of the long distance communicating agents, which are the hormones. The fabrication of these agents are stimulated by hypothalamus. The secreted hormones like oxytocin help the uterine contractions and pain relief [14]. As it is to beexpected, it is important to note that the limbic system, recognized within the InformationalModel of Consciousness as a site of the emotional processes leaded by IES, influences also the endocrine/autonomic nervous system, playing a role in sexual arousal. Within the Informational Model of Consciousness, GTS is connected and acts as a matter-related (genetic) info-output.

The activities of MIS are mainly executed by the brainstem, composed by midbrain, pons and medulla oblongata (or medulla), representing only about $2.6 \%$ from the total weight of the brain [14], which assures a relay-type transit (motor commands to the body and sensory information from the body to the brain) and signals info-integration. The brainstem is connected to spinal cord and with hypothalamus, assuring the main automatic/autonomic vital functions of the organism, concerning the digestion and cardio-respiratory processes like blood pressure/vasomotor activity, cardiac rhythm/rate, vomiting and sneezing, supported especially by medulla. The pons control the signals associated with sleep, respiration, swallowing, bladder activity, hearing/ equilibrium, taste, eye movement, facial expressions/sensation and posture, while midbrain the vision, hearing, motor control, sleep/ wake, arousal/alertness signals and temperature regulation [15]. Some functions of hypothalamus controls and integrates autonomic activities like body temperature, hunger/thirst, fatigue, sleep, and circadian rhythms, attributed to MIS.

The midbrain acts mainly as a helping relay-type transmitter of a part of sensory signals like vision and hearing, motor control, sleep/wakefulness cycle, alert signal sensitivity, temperature regulation and is a prime form of the brain, present even at the ancient vertebrates [16]. As its"substantia nigra" fabricates dopamine, a neurotransmitter involved not only in excitation/ movement and motivation/habitude at human and even to some inferior species like insects, but also in the "happiness"/pleasure/ satisfaction sensations, defined within the Informational Model of Consciousness as "emo-states", this function could be associated mainly to IES and collaterally to GTS due to the dopamine release during the sexual act/gratification and also to IGG, due to the agingassociated decreasing of the dopamine secretion. The expected sensation of satisfaction acts therefore as a reward/motivation for the initiation of some movements/actions. The deficiency of dopamine in the brain is the cause of degenerative Parkinson's disease, while the dysfunction of dopamine activity with respect to the cell receptors is involved in schizophrenia, attention deficit hyperactivity and bipolar disorder, Tourette syndrome (stereotype tics and movements) and addiction. 
IES is mainly related with the limbic system, composed by thalamus, hypothalamus, hippocampus, midbrain and amygdala, responsible for the emotional activities. The limbic system also interacts with the basal ganglia. The basal ganglia, located near the thalamus and hypothalamus transmits/administrates the signal commands from cortex to the motor elements, controlling also the posture and movement. In terms of Informational Model of Consciousness, IES is an essential participant to attitude, which is the info-output of the Operative Informational System (composed by CASI+CDC+IES) for the short/medium-term adaptation, intervening in the posture, as a body external reactive expression of the internal decision. Amygdala is one of the most important component of this system, with functions concerning first of all the detection and reaction to the fear. Amygdala is therefore deeply involved in emotional processes, able to participate in detection/evaluation of danger, including its perception and the strengthening of spatial memory and learning in the adult hippocampus, as emotional experiences. An abnormal function of amygdala induces therefore mental anxiety diseases. Due to its main task, the detection and rapid reaction against the risk/ danger, amygdala plays an important role in attention [17] and the rapid evaluation, within a process which is usually known as the "first impression" [17]. Some functions of hypothalamus refer to parenting attachment behaviour[18], associated to GTS.

Due to its central position between the cortex (which is mainly the headquarter of the analysis and decision within $\mathrm{CDC}$ ) and brainstem/hypophysis, the limbic system is linked to various functions like emotions, sensory (olfaction) processing and action/motor driving, attention/consciousness, autonomic regulation, instincts, memory, but also to dysfunctions like epilepsy, anxiety, bipolar syndrome, schizophrenia [19] Therefore, within the Informational Model of Consciousness, the info-emotional processes are associated to impulsive actions, due to the close/ heavy relation of the amygdala with the reactivity/motor executing elements, initiated by the input information.

The cortex is the outer part, superior level of the human and mammals cerebrum, showing a dense folded area of about 0.12 square meters and 2-4 $\mathrm{mm}$ thickness, which determines a high efficiency of occupation in the cranial space and represents more that $80 \%$ from the brain mass, with about $14-16$ billions of nervous cells [20]. The cortex is the representative area where the thought is active and is the center of the information processing, attention, creativity, decision-making and communication, intelligence, judgement, planning, voluntary control/command of the muscles, specific to CDC. For this reason, within the Informational Model of Consciousness, the data field consisting in values/criteria [21, $22]$, abstract and non-abstract concepts acquired or created on the basis on the existing experience/models stored in CASIand processed by $\mathrm{CDC}$, was defined as the info-creational field, specific to the personal mind universe. This was also the reason to define by the personal representative "I" (myself) the projection into the conscious mind of the informational sub-systems as: CASI =>I know, $\mathrm{CDC}=>$ I want, IES $=>$ I love, MIS $=>$ Iam, GTS $=>$ Icreate, IGG $=>$ Icreated and IC $=>$ Ibelieve. The last definition shows that IC is an antientropic pole of the organism, because this operates as a selective process, reducing from various uncertainty possibilities only one, as a certain information, reducing therefore the entropy of the analysed assumed info-system $[23,1]$.

In order to detect an information in this field, the thought acts as an informational vector/operator, needing an address where this should go $[10,5]$. In the prefrontal cortex is stored the momentary received information from the external/internal sensors, while the long-term consolidated information is deposited/stored in the hippocampus, brain areas specific to CASI. This subsystem is connected to internal and external sensors and the associated circuits, from which the signals are received and perceived. Most sensory information attains the cerebral cortex by means of thalamus, excepting the olfactory signals. Within the Informational Model of Consciousness, the attitude, as a decisional information generated by $\mathrm{CDC}$, is the informational output of the informational system of the human body [5,24], mainly expressed by (but not limited to) the vocal system. Posture and facial expression also contribute to the external display of the internal decision.

As a part of CASI, the cerebellum is the brain component responsible for the long-term memory of the acquired skills and abilities, like the playing an instrument or driving a car. These types of information include besides the memory of theoretical concepts the motor abilities, all of them integrated into unique compact infomotor series of acquired info-executive pathways. The cerebellum is therefore involved in the complex learning processes. Within the Informational Model of Consciousness, the muscles and other similar components of the body are execution/transducers elements [1].

Believed to be only a center of motor controls, coordinating the precision/equilibrium/posture as a fine relay and integration of the movement signals received from the spinal cord, cerebellum is actually an important part not only of the human brain, but also a main constituent of the vertebrate brain. However, at human the cerebellum, with a contribution of about $13 \%$ from the brain weight together with brainstem, is implied not only in infomotor learning, but also in additional functions like attention, language and emotional fear/pleasure regulation [25], indicating it as a valid partner of IES.A special discussion should be done concerning IC center. As specified above, this is an antientropic pole of the informational system of the human body, defined in terms of information as an antientropic center, able to decrease the informational entropy (so of uncertainty) consequently to increase the level of certainty by info-acquirement, which has a key role in the equilibrium of the organism [6].

According to the Informational Model of Consciousness, this pole could be connected to the entropic/antientropic field of matter/ antimatter-like system, explaining the phenomena associated with Near-Death Experiences (NDEs) [26,27] and other extra- 
power properties of the mind like premonition[28]. In terms of neuroscience, this pole is attributed to the anterior cingulate cortex, with a demonstrated implication in Religious and Mystic Experience $[29,30]$. The cingulate structure is a subcortical layer with various functions implied both in conscious (attention and associated eyes motor, anticipation, decision-making, ethics and morality, emotions and especially errordetection[31])and autonomic activities (blood pressure and heart rate) [32], empathy,cooperation, competition, as well as disorders of social cognition [33], constituting practically a managing administrator of information in double senses, from the inferior to the superior direction of the brain and in a reverse order [34].

A summary of this analysis is schematically shown in (Figure 1 ), where the discussed areas of the brain (central side) are represented in correlation with the corresponding informational subsystems of the human body, as defined by IMC (left side). The two columns in the left side of this figure indicate the conscious (left column) and unconscious (right column) components of the informational system of the human body. Within IMC, it was defined as the Operative Informational System (OIS) the sum CASI + CDC + IES, with functions dedicated to info-relation with environment and body for suitable adaptation, and as Programed Informational System (PIS) the sum MIS + GTS + IGG, dedicated to info-management of the metabolic and genetic processes, automatically performed. PIS assures the body maintenance (MIS) and the processing of the genetic input/output information by IGG/GTS respectively. IC is a special automatic body/information and info-social connector/ mediator, as it will be shown also in more details below.

From the perspective of the Informational Model of Consciousness, the brain hierarchical evolution/organization shows an informational-assisted progressive development from simple/automatic functions/structural organization to complex/ info-decisional structures (right side arrow in Figure 1), and the info-integration by epigenetic processes from complex brain infofree decisional structures/areas towards automatic/autonomic zones of the brain, determining the acquirement of new traits and the genetic adaptation to the external cues (left side arrow in Figure 1 ), as it is discussed in more details below.

\section{Discussion}

\section{Informational-assisted Brain Organization and Evolu- tion The neuro-properties of the Info-Connection center}

The experimental findings mentioned above on the activity of the anterior cingulate cortex can be related with the previously defined properties of IC center, as informational/antientropic pole of the organism within the Informational Model of Consciousness (IMC). This correlation shows that this implied brain area intervenes in the evaluation/decision of conflicting situations for the selection of a certainty solution, by the elimination of the error. This is actually the definition of information, discussed earlier within IMC, taking into account that an quantity of information is obtained by the elimination/reduction of uncertainty from an analyzed system
$[3,6]$. Within such a system, the conflicting data represents actually the YES/NO possible alternatives, cingulate cortex deciding in the favour of the most suitable/convenient solution, according to the organism central pro-life objective, i.e. adaptation for survival. These findings justify therefore the IC concerned functions, stipulated by IMC, and its properties claimed earlier $[10,26,28]$ : antientropy (which is favourable to life, against the entropic tendency associated to matter systems), anti-anxiety/equilibrium (conciliation between the contrary opponents), trust (belief in a correct solution by error elimination), security and protection (moderation of pain/fear/distress), peaceful sensation (mediation between amygdala-the alarm center-and the prefrontal awareness cortex), power of multi-events detection (enhancement of the focused/motivated attention and of the view acuity/perception), anticipation (premonition capacity), right judgement (ethics and morality implication), positive orientation of the social relations (empathy stimulation), as claimed within IMC, and even "divine" states experienced by religious practices and evocations [29], asit was shown recently about the Religious and Mystic Experiences, explained by IC-IMC concepts [30]. New experimental evidences on the properties of the anterior cingulate cortex predicted by IC-IMC $[10,23,28]$ were recently reported [35], strongly supporting the IMC and its predictions, mentioned above.

One interesting future investigation line should be specifically dedicated to check if the cell microstructure of the cingulate cortex and their functions could be compatible with the quantum assisted processes described within IMC (mind disembodiment and external exploration) and with the quantum-based model proposed earlier [36]. On the other hand, we have to note that on the evolution scale, the cingulate cortex is a novel structure, very well represented and implicated in the human brain functions, as this analysis shows. Formed bydistinct neuronal, named spindle cells, indicating that this layer is recent on the evolutionary scale [37], this should play an important (maybe a fundamental) role in the accelerated human brain specific adaptive evolution and in the acquirement of new brain features and functions.

\section{Hierarchical info-integration process}

In (Figure 1) is represented in the left side the increase up/ down sense of the integration process of information by means of the informational subsystems of the organism. This is based on the observation concerning the evolution of received/perceived information by CASI, which is initially non related with body informed matter, but progressively included in the intimate cells mechanisms as a consequence of multiple interactions with them. Therefore, within IMC it was distinguished between virtual (non body matter-related) and matter-related information, as interacted with the body cells, by means of epigenetic mechanisms [4].

During the first stage of info-perception from the external sensors and electro-chemical transmission by neuron and interneuron synapses, the information in CASI follows first of all the acquisition step in the short-term memory (frontal cortex), where 
this information cannot be stored for more than a few minutes (typically 1 minute or less). The interaction/"binding" of information with matter during this process is therefore fragile, supported by metastable reversible inter-related mechanisms. During a second step however, which consists in a repetitive/intensively expressed/ addressed information, this can be redistributed and stored in the long-term memory (hippocampus). Therefore, besides the contribution of CASI (perception, transmission and storing process), CDC plays an important role, deciding that this information it is, or not important for own system necessities. Within this phase, the repeatability and/or the intensity of the informational signal with respect to its significance for the security/wellbeing/survival of the organism plays a key role in the long-term memorization. For this, the traumatic (intensively recorded) experiences especially by children, whom the capacity of control is still fragile, are deeply stored in memory, affecting negatively the entire life of the traumatized persons [38].

Therefore, as the informational system of the human body is equipped with an alarm system-amygdala-a part of IES, not always CDC alone could make a decision, specifically in exceptional situations. IES is therefore an engaging partner for integration of a new information within the information system of the human body, not only in special moments/situations, but also during the integration of a certain information implying affectivity. According to IMC, emotions represent actually a body response to the received information, automatic or not, and are stored in CASI too. Amygdala takes automatic decisions and commands, known and expressed by the famous paradigm "fight or flight", transmitting them to the motor elements [20]. Emotional participation for a deep Memorization under a stable and consolidated form is therefore an amplification factor for a deeper integration of information.

By such processes, informed matter, defined within IMC as any component of the body participating to the reception/transmission of information, acquires a new information for adaptation of the body itself under the new ambient/external conditions, for survival. The mechanisms of the information transference between various interacting components of the body multi-particle system were defined as embodiment/disembodiment of information, understanding by this that information, as an immaterial (action/ change) operator, is incorporated or released into or from body matter itself during the interaction process between information and matter [5].A more complex and complete info-integration step consists in the acquisition of the learned info-motor stereotypical behaviours converted into personal abilities, like playing an instrument or driving of a car. Such automatic info-motor sequences are assimilated with the participation of the corresponding motormemory component of the brain - cerebellum. The acquisition of a typical info-motor sequence, becoming by repetition a standard behaviour, seems to be from the evolutionary point of view a precursor of the automatic/autonomic basic processes, driving the body motor vital functions, managed by MIS.
The epigenetic integration process is the ultimate step of infointegration, ready to be transmitted as a genetic information to the offspring. As a consequence of such a process, the new acquired information induced by the external insistent/repetitive cues, is finally codified as acquired traits by the epigenetic core of the cells, without affecting their genetic structure. This process is according to IMC operated by GTS and assumed by IGG of the offspring.The info-integration process described above, suggests therefore that the human brain is able to assure it's own and body evolution for adaptation, by hierarchical up/down progressive incorporation of the external received information, perceived as a stress/cue maintained for a long time period, repetitively/intensively applied, and to transmit it to the next generation for the survival continuity. This progressive info-incorporation is successfully finalized by epigenetic codification of the new acquired information into the genetic core of the cells, without affecting its stable structure. The paradigm "function makes the organ" seems to work by means of such intimate mechanisms with implicit participation of information, as an imprescriptible component. Information is therefore a key/fundamental agent directly implicated in the brain/ body evolutionary process. This process starts from the superior level of the brain, able to select the information, and finalizes with the stable info-integration, following a well-defined trajectory, from CASI-CDC to GTS-IGG via IES and MIS type steps, with the participation of IC (selective antientropic function), as it is shown in the left side of (Figure 1).

\section{The brain hierarchical evolution/organization}

The brain evolution and organization could be therefore discussed as a reverse process, from simple to complex structure, where information is an essential participant and active operator for body and functional evolution. This is shown in (Figure1) right side, where the scale indicative arrow is oriented from basic (lower) to highly organized (upper) structure. As it was remarked above, the basic structure of the brain is the spinal cord, continued by brainstem, cerebellum and limbic system, with fundamental functions consisting in relay-type transmission from andtoward the body, intervening in learning, in time/spatial distribution to the various superior zones of the cortex for info-processing and decision. As the survival is the pivotal objective of the organism, assuring its conditional existence face to the natural conditions, this basic simpler structure is equipped with essential vital tools: the maintainer of the present life operating processes (brainstemMIS + reactive security adviser (amygdala or precursors [39]-IES) + learning tool for adaptation (cerebellum-CASI), and reproduction manager for future life continuity (hypophysis-GTS). By abbreviated names of the informational subsystems indicated in this context, we have to assume only the reduced, primary functions of their activity, completed only if the participation of the superior echelons of the brain, i.e. cortex and cingulate cortex are taken into account. Even so, such a reduced brain system would be auto-sufficient to assure its existence and to affront the living conditions, fulfilling the survival objective. 
The autonomic/automatic working of the most part of such a reduced rudimentary system is by it itself an argument to consider such system a basic, primary form of brain, assuring the minimal but vital functions on the evolutionary scale. Indeed, if we analyse such systems in terms of information/entropy balance, we have to note that these systems dispose of small possible unoccupied states, so of a small capacity for the processing of a new information, because most of them are involved ("occupied") already in automatic chaintype routinist processes. The brain composition of the vertebrates is quite similar with a "reduced" brain, showing a common underlying sub-structure, which appears during the early stages of embryonic (IGG managed) development, before the cortex hemispheres, adapted themselves to the specific necessities within the own living ambient - air, water or land areas [15]. This shows that the brain hemispheres are an adaptable structure, organized latter, upon a basic/primary existing substrate. The embryonic development is actually a quickly-shown movie of the species evolution, nature seems to follow again its learned lesson during a large period of time. In the earlier stage of the development, the brain of vertebrates consists in three distinct structures: hindbrain (medulla, pons and cerebellum), midbrain and forebrain (brain hemispheres), so the minimal basic structure on the evolutionary scale, assuring the brain connection with the body and its vital processes, seems to be the hindbrain. This was confirmed in the case of some very ancient precursors of vertebrates [40]. On the evolutionary scale, IGG is able to configure the anatomic structure and to transmit it by means of GTS to the successors, progressively enriched with the new acquired traits by epigenetic mechanisms [41], successively consolidated and amplified during the lifetime of many generation, if the stress/cue factors are constantly/repetitively maintained [4].

The down/up hierarchical organization of the human brain on the evolutionary scale (Figure 1 right side), from simple to complex structures, starting with basic brainstem assigned to vital automatic functions (MIS) to cerebral cortex (CDC-CASI) via limbic system (IES), is also demonstrated by the cingulate cortex structure (IC site), formed by specific spindle-cells type neurons close to the limbic system, novel on the scale evolution. These cells are able, among many other functions, to detect emotional external feelings [36]. The mothers for instance are sensitive to the child signals by means of these neuronal structures, showing their capability to participate in the inter-human empathic communication. Within IMC, it was shown that this center is implied in the quantum-assisted detection of special range of phenomena, like that associated with NDEs [26], RMEs [30] and PSI (paranormal) experiences [28], as mentioned above.

The input information from sensors is received in cortex in a hierarchic way, from down to upper side, excepting the visual information, which was extensively developed at humans more than other senses like smell, ones they were able to walk on two legs. Even so, the visual signals for face recognition are still sent to amygdala near-instantly for rapid evaluation. The smell information, highly developed to animals, especially to dogs, overtaken at humans by sight, is managed by the limbic system, showing its very ancestral connection with the survival objective. Mechanical vibrations of the sound are transformed in electrical signals and transmitted to brainstem and auditory cortex via thalamus. Together with smell, taste has a survival value, allowing the food selection, within an informational down/up (automatic/decisional) hierarchical infocircuit starting from medulla to cortex via thalamus [36].

The brain areas corresponding to the informational subsystems are connected with body by spinal cord and the nervous ramifications of the nervous system [42]. Pain is a primary signal of danger, fundamental in the body surveillance. Following a down/ up hierarchical scale direction, the pain signals alert the brain on a body injury by pain-transmitting nerves present in almost every region, in a circuit starting from the spinal cord area of the brain, passing from amygdala to various cerebral cortex areas and anterior cingulate cortex, for analysis and motor decision [36].The increasing volume occupied by the brain in the cranial cavity from medulla to cortex as indicated by the conic blue profile in (Figure 1) is an indication of the increased quantity of the neural cells implied in the operation/processing of information. The volume proportions increases from down to upper side on the hierarchical scale as follows: cerebral cortex $77 \%$, midbrain $4 \%$, cerebellum $10 \%$, spinal cord $2 \%$, contrasting with $35 \%$ of the rat spinal cord and $31 \%$ of its cerebral cortex [43]. Careful autopsy determinations show that the human total brain weight is about $1.5 \mathrm{~kg}$, the cerebrum accounting for $87 \%$ of the total brain weight, the cerebellum and brainstem for $13 \%$, whereas the contribution of the attached upper spinal cord is practically negligible $(<2 \mathrm{~g})[44]$.

Looking from the informational point of view to the neural structural organization/evolution of the brain, we have to observe the increasing of the informational potential from the basic autonomic/automatic (PIS) to the complex free-will structures of the cortex (OIS), as shown also by the up-oriented arrow in the right side of Fig. 1. Indeed, in an ideal solid state structure, were the atoms are regularly located in a symmetrical lattice cell repetitively reproduced within the entire structure, the quantity of information is reduced taking into account the small quantity of the necessary states useful to obtain the atom regular repartition $[45,46]$. Therefore, the diversity multiplies the possible states participating to the entropy/information balance, so the infoprocessing capability, as it is the case of the neuronal system of the cerebral cortex. The accelerated brain development of the human species could be regarded therefore as a consequence of two main factors:

(1) Without sufficient force or other physical performance abilities, the unique tool for adaptation was the training of the intelligent (info-processing) structure, by new info-acquisition. 
(2) The antientropic activity of the anterior cingulate cortex, a novel acquired structure in the human brain, favourable to life and its attributes, contributed to this acceleration. The acquisition of new down/up anatomic/function "layers" was / is assisted by information, as discussed above.

On the other hand, as the down-oriented arrow in the left side of (Figure 1) shows, the integration up/down of a selected information acting constantly/insistently as a stress/cue from theambient, is integrated into the stable informational structure of the cell by means of epigenetic mechanisms, adding to human's new genetically transferable traits, so new information. That means in other words that information is able to intervene in the informedmatter structure modifying it accordingly, adding new possible informational states to the system.

\section{Conclusion}

On the basis of the recent reported results on the Informational Model of Consciousness and bipolar info-matter structure of the organism, a careful and detailed analysis is given concerning the informational system of the human body, the specific functions and corresponding brain areas of its main informational components defined as CASI (Center of Acquisition and Storing of Information), CDC (Center of Decision and Command), IES (Info-Emotional Center), MIS (Maintenance Informational System), GTS (Genetic Transmission System), IGG (Info-Genetic Generator) and InfoConnection, which shows that these subsystems are organized in a specific anatomic and functional hierarchical architecture.

In the frame of this analysis, a special attention is focused on the activity of amygdala, as a main contributor of IES and on anterior cingulate cortex, finding that its functions fit very well the properties of the Info-Connection center, as stipulated within the Informational Model of Consciousness, strongly supporting it. Indeed, this center is a moderator of the contrary conflicting concepts and actions, i.e. between YES/NO contrary components of the informational unit, eliminating/reducing the errors in the favour of an equilibrated state of the organism. In other words, this center is active in assuring a favourable state of certainty, reducing in terms of information the entropy and increasing the certainty, inspiring confidence and trust. This remarkable property is manifested also by the intervention in the balance between the contrary emotional states, reducing the fare and anxiety initiated by amygdala and even the pain, offering mind-controlled alternatives. The relation with amygdala, which detects with anticipation (before the conscious cortex) the alarming information, could explain some premonition perceptions, intervening also in the "first impression" type evaluation. A special feature attributed to this center is the emphatic info-communication between mother and children, and the emphatic inter-human social communications. Taking into account the matter/antimatter-like properties introduced by IMC to explain the quantum-assisted special phenomena like NDEs, a new and promising gate for further investigations is open both from the neuroscience/neurology and physics/information science.
Representing on a hierarchical scale the info-integration process by epigenetic mechanisms, it was shown that information is progressively integrated by the brain informational subsystems from the superior informational levels of the brain (CDC, CASI) to the inferior automatic/autonomic levels, regarding both the conscious/ unconscious degree (from OIS to PIS components) and the brain up/down organization/architecture. That means, the insistent cue external (virtual) information for adaptation perceived by CASI and operated by CDC is integrated by multicellular epigenetic processes starting from unstable to stable configuration, and genetically transmitted by GTS to IGG of the new generation.

Taking into account a reverses down/up evolution/organization hierarchical scale in the same representation, it is argued that the brain evolution is/was actually assisted by information, as a revers process. That means, the automatic/autonomic functions were progressively assumed under stress/cue external conditions by some rudimentary structures (brainstem, limbic system, cerebellum), during large-time phases of repetition/epigenetic process-practicing, evolving to the much more complex structure and functions represented by cortex. The accelerated development of human as species during this evolution, un ununderstood reasonably issue up to date, can be explained both by the unique support-intelligence as a tool for adaptation, and the antientropic/ organizing-help info-contributing activity of the cingulate cortex.

\section{Conflict of Interest}

No Conflict of interest.

\section{Acknowledgment}

To my son Adrian and his family, to my daughter Ana and his family.To the Romanian Academy and to the personalities involved in the promotion of this domain.With grateful thanks to Prof MD Sultan Tarlaci, Prof Dr Massimo Pregnolati, Prof Stephan Shafer, Prof Dr Dick Meijer, Prof Dr Dean Radin, Dr Constantin BuluceaHonorary Member of Romanian Academy, Dr Cornel Cobianu, Member of the Academy of Romanian Scientists for stimulating discussions and support. Special tanks to Prof MD Stuart Hameroff for his contributing support of this domain.Special thanks to the Editorial Board of this Journal for the professional support and invitation.

\section{References}

1. Gaiseanu F (2020) Information-Matter Bipolarity of the Human Organism and Its Fundamental Circuits: From Philosophy to Physics/ Neurosciences-Based Modeling. Philosophy Study 10(2): 107-118.

2. Gaiseanu F (2019) Information: from Philosophic to Physics Concepts for Informational Modeling of Consciousness. Philosophy Study 8(8): 368-382.

3. Gaiseanu F (2018) Informational Model of Consciousness: From Philosophic Concepts to an Information Science of Consciousness. Philosophy Study 9(4): 181-196.

4. Gaiseanu F (2019) Epigenetic Information-Body Interaction and Information-Assisted Evolution from the Perspective of the Informational Model of Consciousness. Archives in Biomedical Engineering \& Biotechnology 2(2): 1-6. 
6. Gaiseanu F (2019) The Informational Model of Consciousness: Mechanisms of Embodiment/Disembodiment of Information. NeuroQuantology 17(4): 1-17.

7. Gaiseanu F (2020) Fizica Constiintei si a Vietii: Modelul Informational al Constiintei-Informatia in Neurostiinte, Biocomputere si Biosisteme (in Romanian); (Physics of Consciousness and Life: Informational Model of Consciousness-Information in Neurosciences, Biocomputers and Biosystems), Globe Edit (OmniScriptum International Group, Germany).

8. Walker SI, Davies PCW (2012) The Algorithmic Origins of Life, Journal Royal Society Interface.

9. Draganescu M (1979) Profunzimile lumii materiale (in Romanian) Bucuresti: Editura Politica. (The Depth of the Material World Bucharest, Ed Politica).

10. Draganescu M (1990) Informatia materiei (in Romanian) Bucuresti: Editura Academiei Române (Information of matter, Bucharest, Ed Romanian Academy).

11. Gaiseanu F (2016) Consciousness as Informational System of the Human Body. Consciousness and Life Physics, Cosmology and Astrophysics Journal 16(1): 14-25.

12. Melmed S (2011) The Pituitary ( $3^{\text {rd }}$ edn.) San Diego, CA (USA): Academic Press is an imprint of Elsevier pp: 23-25.

13. Zhang Y, Kim MS, Jia B, Yan J, Zuniga Hertz PJ, et al. (2017) Hypothalamic stem cells control ageing speed partly through exosomal miRNAs. Nature 548: 52-57

14. Herholz SC, Coffey EBJ, Pantev H, and Zatorre RJ (2016) Dissociation of Neural Networks for Predisposition and for Training-Related Plasticity in Auditory-Motor Learning, Cerebral Cortex 26: 3125-3134.

15. Patestas AM and Gartner PL (2006) A textbook of neuroanatomy, Blackwell Publishing Ltd.

16.Zhang J (2019) Secrets of the Brain: An Introduction to the Brain Anatomical Structure and Biological Function: 1-34.

17. Dispenza J (2008) Evolve your brain: The Science of Changing Your Mind. Health Communications, Inc Deerfield Beach, Florida (USA).

18. Gaiseanu F (2019) Destiny or Free Will Decision? A Life Overview from the Perspective of an Informational Modeling of Consciousness Part I: Information, Consciousness and Life Cycle. Gerontology \& Geriatric Studies 4(1): 1-7.

19. Kheirbeck MA, Hen R (2011) Dorsal vs ventral hippocampal neurogenensis: Implications for cognition and mood. Neuro psychopharmacology 36(1): 373-374.

17. MacLean PD (1952) Some psychiatric implications of physiological studies on frontotemporal portion of limbic system (visceral brain). Electroencephalography and Clinical Neurophysiology 4(4): 407-418.

18. Clifford BS, Thomas ES, Jun L (2005) Hypothalamic regulation of sleep and circadian rhythms. Nature 437 (7063): 1257-1263.

19. Iversen SD (1984) Recent advances in the anatomy and chemistry of the limbic system. Psychophannacology of the Limbic System, Oxford University Press, USA, pp: 1-16.

22. Gaiseanu F (2018) Destiny or Free Will Decision? A Life Overview from the Perspective of an Informational Modeling of Consciousness Part II: Attitude and Decision Criteria, Free Will and Destiny. Gerontology \& Geriatric Studies 4(1): 1-7.

23. Gaiseanu F (2019) Language Patterns and Cognitive-Sentient Reality: Certainty/Uncertainty in Cognitive-Sentient Exploration of Reality, Chapter in Media Models to Foster Collective Human Coherence in the PSYCHecology,(edn.) Stephen Brock Schafer, IGI Global, USA.

24. Gaiseanu F (2019) Informational Mode of the Brain Operation and Consciousness as an Informational Related System, Archives in Biomedical Engineering \& Biotechnology: 1(5): 1-7.

25. Schmahmann JS, Caplan D (2006) Cognition, emotion and the cerebellum, Brain 129(2): 290-292.
26. Gaiseanu F (2017) Quantum Assisted Process of Disembody Under Near-Death Conditions: An Informational-Field Support Model. NeuroQuantology 15(1): 4-9.

27. Gaiseanu F (2018) Near Death Experiences and Immortality from the Perspective of an Informational Modeling of Consciousness. Gerontology \& Geriatric Studies 2(3): 1-4

28. Gaiseanu F (2017) An Information Based Model of Consciousness Fully Explaining the Mind Normal/Paranormal Properties. NeuroQuantology 15(2): 132-140.

29. Inzlicht M, Tullett A, Good M (2011) The need to believe: a neuroscience account of religion as a motivated process, Religion, Brain \& Behavior 1 (3): 192-251.

30. Gaiseanu F (2019) Human/Humanity Consciousness and Universe: Informational Relation. Neuro Quantology 17(5): 60-70.

31. Luu P, Pederson SM (2004) The anterior cingulate cortex: Regulating actions in context" In Posner MI (edn.), Cognitive neuroscience of attention. New York: Guilford Press, USA.

32. Thomasine K, ONE Function for the Anterior Cingulate Cortex and General AI: Consistency Curation, Paul Scherrer Institute, CH-5232 Villigen PSI, Switzerland.

33. Apps AJM, Rushworth FSM, and Chang WCS (2016) The Anterior Cingulate Gyrus and Social Cognition: Tracking the Motivation of Others. Neuron 90: 682-707.

34. Moss S, Anterior cingulate cortex, Current Editor: Rowshanak Hashemiyoon, sicotests.

35. Monosov IE (2020) Anterior cingulate is a source of valence-specific information about value and uncertainty, bio Rxiv preprint

36. Parker R, Aldridge S, Page M, Parker S (2009) The Human Brain Book-An Illustrated Guide to Its Structure, Function, and Disorders, DK London, New York, Melbourne, Munich, and Delhi.

37. Hameroff S, Penrose R (2014) Consciousness in the universe, A review of the 'Orch OR' theory. Physics of Life Reviews 11: 39-78.

38. Perry PD (1999) The memories of states: How the brain stores and retrieves traumatic experience, in Splintered Reflections: Images of the Body in Trauma By J Goodwin and R Attias (eds.) Basic Books.

39. Vargas JP, López JC, Portavella M (2012) Amygdala and Emotional Learning in Vertebrates - A Comparative Perspective, The Amygdala-A Discrete Multitasking Manager, Barbara Ferry, Intech Open.

40. Ghysen A (2003) The origin and evolution of the nervous system. Int J Dev Biol 47: 555-562.

41. Gaiseanu F (2019) The Silent Voice of Those Who are no Longer: Transgenerational Transmission of Information from the Perspective of the Informational Model of Consciousness. Gerontology \&Geriatric Studies 5(1): 482-488.

42. Gaiseanu F (2019) Informational Neuro-Connections of the Brain with the Body Supporting the Informational Model of Consciousness. Archives in Neurology \& Neuroscience 4(1): 1-6.

43. Swanson WL (1995) Mapping the human brain: past, present, and future, Trend in Neuroscience 18 (11): 471-474.

44. Lindboe CF (2003) Clin Neuropathol 22(6): 263-265.

45. Gaiseanu F (2013) Contributions to the Modelling and Simulation of the Atomic Transport Processes in Silicon and Polysilicon and Applications. Proceedings of the Romanian Academy 4(4): 376-384.

46. Gaiseanu F (2017) Modeling and Simulation of the Impurity Diffusion and Related Phenomena in Silicon and Polysilicon Systems in Microfabrication and Micromachining Technologies. Annals of the Academy of Romanian Scientists, Series on Science and Technology of Information 10(1): 41-78. 